\title{
Perfil clínico e fatores associados ao óbito de pacientes COVID-19 nos primeiros meses da pandemia
}

\author{
Clinical profile and factors associated with the death of COVID-19 patients in the first months of the \\ pandemic \\ Perfil clínico y factores asociados a la muerte de pacientes COVID-19 en los primeros meses de la \\ pandemia
}

Leticia Pontes ${ }^{1}$ (1)

Mitzy Tannia Reichembach Danski ${ }^{1}$ (1) Simone Martins Nascimento Piubello ${ }^{1}$ (D) Jéssica de Fátima Gomes Pereira ${ }^{1}$ (i) Leonardo Bigolin Jantsch ${ }^{2}$ (D)

Letícia Bettega Costa ${ }^{1}$ (D) Juliana de Oliveira dos Santos ${ }^{3}$ (1) Andrea Moreira Arrué ${ }^{1}$ (D)

1. Universidade Federal do Paraná, Departamento de Enfermagem, Programa de Pós-Graduação em Enfermagem. Curitiba, PR, Brasil.

2. Universidade Federal de Santa Maria, Departamento de Enfermagem, Campus Palmeiras das Missões. Santa Maria, RS, Brasil.

3. Universidade Federal do Paraná, Complexo Hospitalar de Clínicas. Curitiba, PR, Brasil.
Autor correspondente:

Simone Martins Nascimento Piubello.

E-mail: snpiubello@gmail.com.

Recebido em 10/05/2021.

Aprovado em 06/09/2021.

DOI:https://doi.org/10.1590/2177-9465-EAN-2021-0203

\section{RESUMO}

Objetivo: analisar as características individuais, clínicas e os fatores associados à mortalidade de pacientes com COVID-19, em hospital público do estado do Paraná, Brasil. Métodos: estudo seccional, retrospectivo, documental $(n=86)$, com pacientes adultos internados, de março a junho de 2020. Resultados: a mortalidade foi de 12,8\%, o grupo de maior risco foi de idosos com comorbidades, especialmente, cardiovasculares. A chance de óbito foi 58 vezes maior em idosos, comparada aos adultos, e oito vezes maior naqueles com comorbidades, comparadas aos hígidos. A maioria dos pacientes apresentou sintomatologia respiratória, febre e mialgia. Tratamento à base de antibióticos, anticoagulantes e antivirais, associado ao suporte ventilatório. As principais complicações foram hipóxia, insuficiência renal aguda e infecção secundária. Conclusão e implicações para a prática: idosos com comorbidades cardiovasculares que necessitaram de cuidados intensivos apresentaram maior chance de óbito. Os resultados de um dos centros de referência na pandemia possibilitam discutir medidas epidemiológicas adotadas com ênfase em conceitos restritivos nos primeiros meses.

Palavras-chave: COVID-19; Estudos Transversais; Morbidade; Mortalidade; SARS-CoV-2.

\section{ABSTRACT}

Objective: to analyze the individual and clinical characteristics and the factors associated with mortality in patients with COVID-19, in a public hospital in the state of Paraná, Brazil. Methods: a cross-sectional, retrospective, documentary study ( $\mathrm{n}=$ 86), with adult inpatients, from March to June 2020. Results: mortality was $12.8 \%$, the highest risk group was the elderly with comorbidities, especially cardiovascular ones. The chance of death was 58 times higher in the elderly compared to adults, and eight times higher in those with comorbidities compared to the healthy ones. Most patients presented with respiratory symptoms, fever, and myalgia. Treatment was based on antibiotics, anticoagulants and antivirals, associated with ventilatory support. The main complications were hypoxia, acute renal failure, and secondary infection. Conclusion and implications for practice: elderly people with cardiovascular comorbidities who required intensive care had a higher chance of death. The results from one of the reference centers in the pandemic make it possible to discuss epidemiological measures adopted, with emphasis on restrictive concepts in the first months.

Keywords: COVID-19; Cross-Sectional Studies; Morbidity; Mortality; SARS-COV-2.

\section{RESUMEN}

Objetivo: analizar las características individuales, clínicas y los factores asociados a la mortalidad en pacientes con COVID-19 en un hospital público del estado de Paraná. Métodos: estudio transversal, retrospectivo, documental $(n=86)$, con pacientes adultos hospitalizados, de marzo a junio de 2020. Resultados: la mortalidad fue del 12,8\%, grupo de mayor riesgo para los ancianos con comorbilidades, especialmente enfermedades cardiovasculares. La probabilidad de muerte fue 58 veces mayor en los ancianos en comparación con los adultos y ocho veces mayor en aquellos con comorbilidades en comparación con los sanos. La mayoría de los pacientes presentaban síntomas respiratorios, fiebre y mialgia. Tratamiento a base de antibióticos anticoagulantes y antivirales, asociado al soporte ventilatorio. Las principales complicaciones fueron hipoxia, insuficiencia renal aguda e infección secundaria. Conclusión e implicaciones para la práctica: los ancianos con comorbilidades cardiovasculares que requirieron cuidados intensivos tenían una mayor probabilidad de muerte. Los resultados de uno de los centros de referencia pandémica permiten discutir las medidas epidemiológicas adoptadas, con énfasis en conceptos restrictivos en los primeros meses.

Palabras clave: COVID-19; Estudios Transversales; Morbilidad; Mortalidad; SARS-COV-2. 


\section{INTRODUÇÃO}

A pandemia causada pela Severe Acute Respiratory Syndrome Coronavirus 2 (SARS-CoV-2) revelou a importância da saúde global. Esse vírus surgiu em um país e se propagou, rapidamente, para outros, provocando crise no sistema de saúde mundial ${ }^{1}$.

No Brasil, o primeiro caso ocorreu em fevereiro de 2020 e, inicialmente, os casos se concentraram nas grandes metrópoles, disseminando-se das capitais aos municípios ${ }^{2}$. Desde o início da doença no Brasil, até o dia 28 de julho de 2021, houve mais de 19 milhões de casos e 551.835 óbitos $^{3}$, sendo considerado o segundo país com maior número de mortes no mundo. ${ }^{4} \mathrm{Em}$ Curitiba, têm-se, 258.679 casos e 6.642 óbitos $^{5}$.

Com os números de infectados e mortos pela SARS-CoV-2 em crescimento exponencial, os cientistas não medem esforços para aprender mais sobre o vírus e a doença Coronavirus Disease 2019 (COVID-19). Compreender como o vírus afeta as pessoas que estão doentes e conhecer o tratamento adequado constituem desafios para todos os países atingidos pela pandemia.

Com o crescimento de casos, uma das políticas públicas utilizadas para o suporte às pessoas com COVID-19 foi o aumento do número de leitos, tanto de enfermarias como de Unidades de Terapia Intensiva (UTI), apesar desse aumento ser insuficiente para demanda da população por serviços hospitalares ${ }^{6}$.

Para que haja um plano de ação de saúde efetivo no combate à pandemia, é necessário sistema nacional de saúde com coleta de dados, quantificando o efeito da doença no setor de assistência à saúde, dados que versem a respeito do número de casos e mortes por idade, gênero, etnia e ambiente de cuidados, estratificado por área de assistência à saúde, como enfermaria e $\mathrm{UTI}^{7}$.

Conhecer as características dos pacientes que foram internados por COVID-19 oferece subsídios aos profissionais de saúde e gestores para elaboração de estratégias para população de maior risco de adoecimento, em virtude das probabilidades de complicações relacionadas à doença e demanda por um leito hospitalar ${ }^{8}$.

Logo, objetivou-se analisar as características individuais, clínicas e os fatores associados à mortalidade de pacientes com COVID-19, em hospital público do estado do Paraná, Brasil.

\section{MÉTODO}

Trata-se de estudo seccional, retrospectivo, analítico que incluiu população de 86 pacientes adultos com COVID-19 e internados em hospital público na Região Sul do país, no período de 26 de março de 2020 e 31 de junho de 2020.

A pesquisa foi desenvolvida em hospital referência para tratamento de pacientes com COVID-19, na capital. Em 23 de março de 2020, a Instituição foi credenciada, pela primeira vez, em centro dedicado a essa doença e, com a aumento da demanda, o hospital disponibilizou unidades específicas para tratamento daqueles pacientes.

Participaram do estudo os adultos que estiveram internados no período de coleta de dados e respondiam aos critérios de seleção estabelecidos. Os critérios de inclusão foram: pacientes adultos hospitalizados, com idade igual ou superior a 18 anos, diagnóstico confirmado para COVID-19, critérios clínicos e resultado positivo para reação em cadeia de polimerase via transcriptase reversa em tempo real (RT-PCR) para SARS-CoV-2 em amostras respiratórias (swabs de nasofaringe e/ou orofaringe).

Excluíram-se do estudo seis prontuários, pois não foi possível localizar o registro após cinco tentativas, impossibilitando a coleta de dados. Do total, de 92 prontuários elegíveis para o estudo, seis foram excluídos, totalizando amostra de 86 prontuários.

No período de elaboração desta pesquisa, havia duas enfermarias respiratórias, I e II, localizadas nos sétimo e nono andares, respectivamente, do prédio central, com total de 52 leitos, e cinco UTI COVID: UTI COVID I e ll que se localizavam no sexto andar; a III, localizada no $12^{\circ}$ andar; a IV, no $13^{\circ}$ andar; e a V, no terceiro andar do prédio anexo, totalizando 63 leitos.

As unidades eram contempladas com equipe multidisciplinar, incluindo, aproximadamente, 71 enfermeiros, 182 técnicos e 23 auxiliares de enfermagem, respeitando o dimensionamento recomendado pelo Conselho Federal de Enfermagem (COFEN).

Os dados dos participantes foram obtidos a partir dos registros em papéis disponíveis no Serviço de Arquivo Médico e Estatística (SAME) e consolidados por meio de instrumento idealizado pelos pesquisadores e desenvolvido com suporte de especialista em tecnologia da informação. Utilizou-se da linguagem Visual Basic ${ }^{\circledR}(\mathrm{VBA})$, no programa Microsoft Excel $97^{\circledR}$. Os dados dos prontuários foram preenchidos pelos profissionais de saúde da referida instituição que prestavam assistência direta ao paciente. Essas informações foram coletadas do prontuário e registradas no instrumento, previamente, testado pela equipe de pesquisadores, composta por duas doutorandas, uma mestranda em enfermagem e uma enfermeira da instituição. A coleta dos dados ocorreu de setembro a novembro de 2020.

O instrumento compreendia, algumas variáveis, sendo destacadas: as condições individuais e clínicas como sintomas prévios, comorbidades (por sistemas do corpo humano), as complicações durante o período de internação, os tratamentos instaurados durante a internação, isto é, os medicamentos (antibióticos, anticoagulantes, corticosteroides, terapia antirretroviral) e o suporte respiratório (cateter nasal, máscara de alta concentração com reservatório, ventilação mecânica, macronebulização). Utilizou-se como desfecho da internação a condição alta hospitalar e óbito, definida no prontuário.

As variáveis independentes, de caracterização social, foram: sexo (feminino e masculino), idade (grupos etários), raça (branco, pardo e negro), procedência (local de residência), estado civil (casado, solteiro, viúvo).

As variáveis categóricas foram apresentadas em números absolutos (n) e percentuais (\%) e analisadas pelo teste do QuiQuadrado para comparação de frequência e odds ratio, além do Teste exato de Fisher. Variáveis contínuas foram representadas com Média $\left(\mathrm{M}_{\mathrm{e}}\right)$ e Desvio padrão $( \pm \mathrm{Dp})$. Para análise dos dados, utilizou-se do software Statistical Package for the Social Sciences (SPSS 22.0) for Windows $^{\circledR}$. As variáveis idade $(<65$ anos, 
$\geq 65$ anos), raça (branca, parda/negra), comorbidade (sim, não) e número de comorbidades (uma, duas ou mais) foram categorizadas para as análises estatísticas. Consideraram-se significativos valores de $\mathrm{p}<0,05$.

Por se tratar de análise retrospectiva, o consentimento informado foi dispensado pelo Comitê de Ética em Pesquisa, considerando que os dados foram obtidos, diretamente, do prontuário clínico, sem utilização de outras técnicas para coleta, como entrevista ou exame físico. O estudo foi aprovado pelo Comitê de Ética em Pesquisa da instituição lócus do estudo, respeitando a Resolução 466/2012, conforme parecer 4.183.502, no ano 2020.

\section{RESULTADOS}

Entre março e junho de 2020, 92 pacientes diagnosticados com COVID-19 foram internados no local do estudo, no entanto seis prontuários foram excluídos. As características individuais e demográficas dos 86 pacientes analisados, no período, estão descritas na Tabela 1.

É possível destacar maior prevalência de óbito em pessoas com mais de 65 anos, da raça branca, provenientes de municípios fora do estado do Paraná (visitantes), viúvos e casados. Ao avaliar as características com o desfecho, as análises mostraram que não houve diferença significativa entre o sexo masculino e feminino $(p>0,05)$.

Em dez prontuários, havia a informação de que o paciente não tinha viajado para fora do país, nos demais, não havia dados sobre viagens internacionais recentes $(n=76)$. Cerca de 17 tinham história de contato próximo com caso positivo de SARS-CoV-2, destes, 16 foram com familiar e um, com outra pessoa. Nove tiveram contato com casos suspeitos, dos quais, oito eram familiares e um colega de trabalho, os demais, 60 prontuários, não tinham informação sobre contato com caso suspeito ou confirmado.

Tabela 1. Caraterísticas individuais e demográficas (n=86). Curitiba, Paraná, Brasil, 2021.

\begin{tabular}{|c|c|c|c|c|}
\hline \multirow{2}{*}{ Variáveis } & \multicolumn{2}{|c|}{ Desfechos } & \multirow{2}{*}{$p$-valor* } & \multirow{2}{*}{ n (\%) } \\
\hline & Alta & Óbito & & \\
\hline \multicolumn{5}{|l|}{ Sexo } \\
\hline Masculino & $44(89,8)$ & $5(10,2)$ & 0,402 & $49(57,0)$ \\
\hline Feminino & $31(83,8)$ & $6(16,2)$ & & $37(43,0)$ \\
\hline \multicolumn{5}{|l|}{ Grupo Etário (anos) } \\
\hline $18-29$ & $7(87,5)$ & $1(12,5)$ & & $8(9,3)$ \\
\hline $30-49$ & $30(100)$ & 0 & & $30(34,9)$ \\
\hline $50-64$ & $27(100)$ & 0 & & $27(31,4)$ \\
\hline$\geq 65$ & $11(52,4)$ & $10(47,6)$ & & $21(24,4)$ \\
\hline Branco & $53(85,5)$ & $9(14,5)$ & & $62(72,1)$ \\
\hline Pardo & $13(100)$ & 0 & & $13(15,1)$ \\
\hline Negro & $7(100)$ & 0 & & $7(8,1)$ \\
\hline Não informado & $2(50)$ & $2(50,0)$ & & $4(4,7)$ \\
\hline \multicolumn{5}{|l|}{ Procedência } \\
\hline Residem no município do hospital (Curitiba) & $50(90,9)$ & $5(9,1)$ & & $55(64,0)$ \\
\hline Região Metropolitana de Curitiba & $19(86,4)$ & $3(13,6)$ & & $22(25,6)$ \\
\hline Outro município do Paraná & $4(100)$ & 0 & & $4(4,6)$ \\
\hline Município fora do Paraná & $1(50,0)$ & $1(50,0)$ & & $2(2,3)$ \\
\hline Não informado & $1(100)$ & 0 & & $3(3,5)$ \\
\hline \multicolumn{5}{|l|}{ Estado Civil } \\
\hline Casado(a)/União estável & $29(80,6)$ & $7(19,4)$ & & $36(41,9)$ \\
\hline Solteiro(a) & $16(94,1)$ & $1(5,9)$ & & $17(19,8)$ \\
\hline Viúvo(a) & $4(80,0)$ & $1(20,0)$ & & $5(5,8)$ \\
\hline Não informado & $24(92,3)$ & $2(7,7)$ & & $26(30,2)$ \\
\hline Total & $75(87,2)$ & $11(12,8)$ & & $86(100)$ \\
\hline
\end{tabular}

FONTE: dados da pesquisa, 2020. *Teste do Qui-quadrado. 
Do total de pacientes internados, 62 (72\%) foram admitidos nas enfermarias e $24(28 \%)$ nas UTI, mas cinco pacientes que estavam na enfermaria, em algum momento, necessitaram de cuidado intensivo e foram encaminhados para UTI, totalizando 29 pacientes internados na UTI. O tempo mínimo de internação nos dois setores foi de um dia, nas enfermarias, o máximo foi de 33 dias $\left(M_{e}=7,6\right.$ e $\left.D_{p}=( \pm) 5,6\right)$ e, na UTI, de 28 dias $\left(M_{e}=9,5\right.$; $\left.D_{p}=( \pm) 8,4\right)$.

Os sintomas prévios, as complicações desenvolvidas no período de internação e os medicamentos utilizados no tratamento estão na Tabela 2.
Os principais sintomas descritos nos prontuários foram respiratórios, como tosse e dificuldade de respirar, seguidos de febre e mialgia. As complicações mais prevalentes: hipóxia, insuficiência renal e infecção secundária e tratamento medicamentos à base de antibióticos, anticoagulantes e antirretrovirais.

Com relação ao suporte ventilatório, aproximadamente, $85 \%$ $(n=73)$ dos pacientes necessitaram de oxigenação. A maioria utilizou cateter nasal $(n=70)$, máscara de alta concentração com reservatório ( $\mathrm{n}=21$ ) e Ventilação Mecânica Invasiva (VMI) $(n=12)$. Apenas um paciente precisou de macronebulização $e$ outro, de traqueostomia.

Tabela 2. Variáveis clínicas e de tratamento medicamentoso. Curitiba, Paraná, Brasil, 2021.

\begin{tabular}{|c|c|c|}
\hline Variáveis & $\operatorname{Sim}(\%)$ & Não (\%) \\
\hline \multicolumn{3}{|l|}{ Sintomas } \\
\hline Tosse & $66(76,7)$ & $22(23,3)$ \\
\hline Dificuldade de respirar & $64(74,4)$ & $22(25,6)$ \\
\hline Febre & $56(65,1)$ & $30(34,9)$ \\
\hline Dor muscular & $40(46,5)$ & $46(53,5)$ \\
\hline Cefaleia & $24(27,9)$ & $62(72,1)$ \\
\hline Ageusia (ausência ou redução no paladar) & $18(20,9)$ & $68(79,1)$ \\
\hline Diarreia & $18(20,9)$ & $68(79,1)$ \\
\hline Coriza & $14(16,3)$ & $72(83,7)$ \\
\hline Fadiga & $14(16,3)$ & $72(83,7)$ \\
\hline Anosmia (diminuição ou perda do olfato) & $13(15,1)$ & $73(84,9)$ \\
\hline Náusea & $10(11,6)$ & $76(88,4)$ \\
\hline Dor de garganta & $9(10,5)$ & $77(89,5)$ \\
\hline Êmese (vômitos) & $9(10,5)$ & $77(89,5)$ \\
\hline Dor abdominal & $4(4,7)$ & $82(95,3)$ \\
\hline Calafrios & $1(1,2)$ & $85(98,8)$ \\
\hline Congestão Nasal & $1(1,2)$ & $85(98,8)$ \\
\hline \multicolumn{3}{|l|}{ Complicações } \\
\hline Hipóxia & $27(31,4)$ & $59(68,6)$ \\
\hline Insuficiência Renal Aguda & $10(11,6)$ & $76(88,4)$ \\
\hline Infecção secundária & $8(9,3)$ & $78(90,7)$ \\
\hline Evento tromboembólico & $3(3,5)$ & $83(96,5)$ \\
\hline Apneia & $2(2,3)$ & $84(97,7)$ \\
\hline \multicolumn{3}{|l|}{ Medicamentos } \\
\hline Azitromicina & $78(90,7)$ & $8(9,3)$ \\
\hline Anticoagulantes & $74(86,0)$ & $12(14,0)$ \\
\hline Dexametasona & $46(53,6)$ & $40(46,5)$ \\
\hline Outros antibióticos e antiviral ( $n=81)$ & $56(69,1)$ & $25(30,9)$ \\
\hline Outros Antibióticos $(n=81)$ & $19(23,5)$ & $62(76,5)$ \\
\hline Antiviral ( $n=81$ ) & $6(7,4)$ & $75(92,6)$ \\
\hline
\end{tabular}

FONTE: dados da pesquisa, 2020. 
Do total de pacientes assistidos no hospital investigado, cerca de $58,1 \%(n=50)$ tinham algum tipo de comorbidade prévia. Destaca-se que 36 pessoas apresentavam duas ou mais comorbidades, e as principais doenças foram: $76 \%(n=38)$ cardiovasculares, $22 \%(n=11)$ neurológicas, $12 \%(n=6)$ pulmonar, $2 \%(n=1)$ hepática e $2 \%(n=1)$ nefrológica.

No que tange às variáveis idade, raça e condições de saúde pregressas, destaca-se a Tabela $3 \mathrm{com}$ a análise dos fatores associados ao desfecho.

Durante os primeiros 128 dias da pandemia, 11 dos 86 pacientes morreram, resultando em taxa de mortalidade de 12,8\%. Destes, $82 \%$ ( $n=9)$ foram a óbito na UTI e 18\% $(n=2)$ na enfermaria. Com relação ao sexo, $54,5 \%$ ( $n=6)$ eram mulheres e $45,5 \%(n=5)$ homens.

O único paciente jovem que foi a óbito, com idade entre 18 e 29 anos, tinha diagnóstico de tumor medular em região cervical, pós-cirurgia para ressecção do tumor. A chance de óbito, na população maior que 65 anos, foi 58 vezes maior, quando comparada aos menores de 65 anos. Outro fator a ser destacado na análise é a presença de comorbidades, visto que a chance de óbito para aqueles com comorbidades foi oito vezes maior, quando comparada à população, previamente, hígida. Destacam-se as doenças cardiovasculares como as de pior desfecho.

Tabela 3. Associação de fatores ao desfecho alta e óbito em pacientes com COVID-19 (n=86). Curitiba, Paraná, Brasil, 2021.

\begin{tabular}{|c|c|c|c|c|}
\hline \multirow{2}{*}{ Variáveis } & \multicolumn{2}{|c|}{ Desfechos } & \multirow{2}{*}{$\mathrm{p}$-valor* } & \multirow{2}{*}{ OR (IC95\%) } \\
\hline & Alta & Óbito & & \\
\hline Idade (anos) & & & $<0,001$ & \\
\hline$<65$ & $64(98,5)$ & $1(1,5)$ & & 1 \\
\hline$\geq 65$ & $11(52,4)$ & $10(47,6)$ & & $\begin{array}{c}58,182 \\
(6,756-500,918)\end{array}$ \\
\hline \multicolumn{5}{|l|}{ Raça } \\
\hline Branco & $54(85,7)$ & $9(14,3)$ & 0,720 & $\begin{array}{c}1,750 \\
(0,349-8,781)\end{array}$ \\
\hline Parda/Negra & $21(91,3)$ & $2(8,7)$ & & 1 \\
\hline \multicolumn{5}{|l|}{ Presença de comorbidade } \\
\hline Sim & $40(80,0)$ & $10(20,0)$ & 0,018 & $\begin{array}{c}8,750 \\
(1,066-71,818)\end{array}$ \\
\hline Não & $35(97,2)$ & $1(2,8)$ & & 1 \\
\hline \multicolumn{5}{|l|}{ Número de comorbidades } \\
\hline Uma & $22(91,7)$ & $2(8,3)$ & & 1 \\
\hline Duas ou mais & $27(75,0)$ & $9(25,0)$ & 0,095 & $\begin{array}{c}3,667 \\
(0,717-18,758)\end{array}$ \\
\hline \multicolumn{5}{|l|}{ Tipo de comorbidade } \\
\hline \multicolumn{5}{|l|}{ Neurológica } \\
\hline Sim & $8(72,7)$ & $3(27,3)$ & 0,145 & $\begin{array}{c}3,141 \\
(0,690-14,302)\end{array}$ \\
\hline Não & $67(89,3)$ & $8(10,7)$ & & 1 \\
\hline \multicolumn{5}{|l|}{ Cardiovascular } \\
\hline Sim & $29(76,3)$ & $9(23,7)$ & 0,009 & $\begin{array}{c}7,138 \\
(1,440-35,393)\end{array}$ \\
\hline Não & $46(95,8)$ & $2(4,2)$ & & 1 \\
\hline \multicolumn{5}{|l|}{ Pulmonar } \\
\hline Sim & $5(83,3)$ & $1(16,7)$ & 0,572 & $\begin{array}{c}1,400 \\
(0,148-13,242)\end{array}$ \\
\hline Não & $70(87,5)$ & $10(12,5)$ & & 1 \\
\hline
\end{tabular}

FONTE: dados da pesquisa, 2020. * Teste Exato de Fisher. 


\section{DISCUSSÃO}

Este é um dos primeiros estudos que explora as características de pacientes com diagnóstico de COVID-19, em hospital referência, nos primeiros meses de pandemia, em Curitiba, Brasil. A pandemia continua sem sinais de remissão, exigindo soluções sistemáticas.

Com o aparecimento dos primeiros casos em Curitiba, no dia 12 de março de 2020, o governo municipal decretou situação de emergência em saúde pública, no dia 16 de março, e declarou a implementação de medidas preventivas e restritivas, como suspensão de eventos abertos ao público, de qualquer natureza, com aglomeração acima de 50 pessoas, instituição de teletrabalho, cancelamento da circulação de transporte coletivo rodoviário interestadual de passageiros com origem de todas as unidades federativas do país e do Distrito Federal, fechamento de shopping centers, galerias e estabelecimentos congêneres, academias ou centros de ginásticas, instituições escolares e universidades, entre outras. Além de medidas de distanciamento social, higienização das mãos, etiqueta da tosse e uso de máscaras ${ }^{10}$. A taxa de incidência da infecção em moradores de Curitiba foi a menor entre as dez capitais mais populosas do Brasil, nos primeiros dois meses de pandemia ${ }^{11}$.

Durante os primeiros 128 dias da pandemia, 11 dos 86 pacientes morreram, resultando em mortalidade de $12,8 \%$, dos quais, $82 \%$ foram a óbito na UTI e $18 \%$ na enfermaria. Indicador que denota baixa prevalência ao comparar com a mortalidade hospitalar geral no Brasil que, no mesmo período, foi de $38 \%{ }^{12}$. Os resultados mostram que os pacientes hospitalizados com infecção por SARS-CoV-2 demandam atenção clínica e intensiva da enfermagem.

O perfil individual e demográfico evidenciou que a maioria dos pacientes era do sexo masculino, raça branca, casado, menor de 65 anos e procedentes de Curitiba e região metropolitana. Estudos realizados, internacionalmente, mostram predominância do sexo masculino e mediana de idade de maior internação em pacientes com menos de 65 anos de idade ${ }^{12-14}$. Coorte realizada no Brasil mostrou proporção maior de pessoas da raça branca na Região Sul, representando 88\% dessa população ${ }^{12}$.

A maioria dos pacientes foi admitida em enfermaria, no entanto, 33,7\%, em algum momento, necessitaram de cuidado intensivo. O tempo médio de internação foi de sete dias na enfermaria e de nove na UTI.

Em estudo com 2.215 casos de UTI nos Estados Unidos da América (EUA), o tempo médio desde o início até a admissão na UTI foi de sete dias ${ }^{15}$. Em coorte realizada no Brasil, houve a mesma média de dias para admissão na UTI ${ }^{12}$. De acordo com estudo de coorte, quase $80 \%$ de todas as admissões na UTI ocorreram nos primeiros cinco dias de internação. Para todos os casos graves, cerca de $40 \%$ foram admitidos em UTI, com $29 \%$ necessitando de $\mathrm{VMI}^{13}$.

A pesquisa evidenciou prevalência de sintomatologia respiratória, febre (65\%), seguido de mialgia (46\%). De acordo com o Ministério da Saúde (MS), os principais sintomas relacionados à COVID-19 são: febre (83\%), tosse (82\%), falta de ar (31\%) e dor muscular $(11 \%)^{16}$. Um aplicativo para smartphone para levantamento de sintomas com mais de 2 milhões de participantes, nos EUA e em Londres, mostrou que os principais sintomas relatados pelos pacientes foram: anosmia e ageusia, somando $64,76 \%$, tosse $(56,73 \%)$, dor no peito $(42,73 \%)$, falta de apetite $(42,03 \%)$ e febre $(34,34 \%)^{1}$.

Os sintomas da COVID-19 podem aparecer em intervalo de dois a 14 dias após a exposição. O período médio de incubação é de, aproximadamente, 5,2 dias. A transmissibilidade dos pacientes infectados é, em média, de sete dias após o início dos sintomas. No entanto, a transmissão pode ocorrer mesmo sem o aparecimento de sinais e sintomas ${ }^{17,18}$.

Os resultados deste estudo mostraram que $42 \%$ dos pacientes tinham duas ou mais comorbidades, em destaque as cardiovasculares, neurológicas e pulmonares. Os pacientes com comorbidades, especialmente, cardiovasculares, tiveram maior chance de óbito.

A Secretaria de Vigilância de Saúde do Ministério da Saúde do Brasil alerta que as principais comorbidades identificadas nos pacientes diagnosticados com COVID-19 são: cardiopatia, diabetes, doença neurológica, doença renal e pneumopatia ${ }^{19}$.

Outro estudo também aponta que pessoas com doenças cardiovasculares têm maior probabilidade de desenvolver complicações graves da COVID-19, incluindo internação hospitalar e morte ${ }^{20}$

Nos EUA, o Centers for Disease Control and Prevention (CDC), mediante o monitoramento em 14 estados, por meio da COVID-NET, ferramenta para monitoramento da demografia de pacientes com COVID-19, mostra que as principais comorbidades relacionadas à hospitalização são doença cardiovascular $(56,3 \%)$, obesidade $(48,2 \%)$ e diabetes $(42 \%)^{21}$.

As complicações mais prevalentes foram hipóxia, insuficiência renal e infecção secundária. A maioria necessitou de suporte ventilatório e $14 \%$ de VMI. Em estudo de coorte, 36\% dos pacientes apresentaram insuficiência renal, sendo a maioria pacientes com VMI e comorbidades ${ }^{22}$.

Nas doenças respiratórias críticas, a VMI é um método de tratamento essencial e eficaz para criação de uma via aérea artificial, mantendo boa ventilação e auxiliando no controle das infecções pulmonares ${ }^{23}$. Em estudo realizado no Brasil, dos que necessitaram de VMI, a taxa também foi de $14 \%$ entre a população estudada ${ }^{12}$.

Quanto ao tratamento utilizado, a medicação mais utilizada foi a azitromicina, seguida do uso de anticoagulante e antiviral em associação com antibióticos.

A azitromicina é um antibiótico usado, principalmente, em infecções bacterianas pulmonares, porém, em estudos, comprovouse que também possui atividade antiviral in vitro, agindo contra o vírus SARS-CoV-2, além de exercer papel imunomodulador, que pode interromper respostas inflamatórias intensas potenciais causadores de progressão para falência de órgãos e morte por COVID-1924. Em estudo realizado em Nova York, essa medicação foi a segunda mais utilizada no tratamento ${ }^{14}$. 
Outro estudo aponta que a taxa elevada de dímeros $D$ foi associada a um pior desfecho, aumentando a necessidade de intubação e complicações trombóticas ${ }^{25}$. Porém, no uso de anticoagulantes, é essencial que o profissional esteja atento ao risco de sangramento que pode ser aumentado pelo uso da medicação.

A mortalidade, deste estudo, foi de $12,8 \%$. Idosos com comorbidades cardiovasculares foram associados ao pior desfecho. Os idosos, que constituem população vulnerável, e pacientes com condições crônicas, como diabetes e doenças cardiovasculares ou pulmonares, não somente correm maior risco de desenvolver doenças graves, como também de ir a óbito pela doença ${ }^{18}$.

O CDC alerta que os idosos correm maior risco de necessitar de hospitalização ou ir a óbito devido ao diagnóstico da COVID-19. Também destaca que pessoas com mais de 65 anos apresentam taxa de mortalidade 90 vezes maior, em comparação com pessoas de 18 a 29 anos. Quando a idade é maior que 85 anos, esse risco aumenta, chegando a ser 630 vezes maior ${ }^{26}$.

As mudanças fisiológicas do envelhecimento e das comorbidades relacionadas à idade, como doenças cardíacas e pulmonares, diabetes, demência e polifarmácia, estão associadas a resultados desfavoráveis em pacientes mais idosos ${ }^{27}$. Segundo estudo, nas populações acima de 60 anos, as comorbidades se apresentam como fator de risco ${ }^{28}$

Portanto, o perfil da população mais associada a óbitos, segundo este estudo, abrange homens brancos, com idades acima de 65 anos, com presença de comorbidade, principalmente, a doença cardiovascular.

Este estudo examinou a pandemia da COVID-19 do ponto de vista de um único centro. Porém, enfatiza-se que a estratégia municipal liderada pelas medidas epidemiológicas imediatas em Curitiba, durante a pandemia, resultou em número relativamente reduzido de pacientes graves e mortes, em comparação com o restante do Brasil, nos primeiros meses de pandemia Outra limitação foi a qualidade dos registros dos prontuários selecionados, uma vez que muitos tinham dados incompletos, fato que dificultou a realização da coleta e análise dos dados. Logo, evidencia-se a relevância do planejamento de medidas que melhorem a qualidade dos registros no prontuário do paciente, bem como o desenvolvimento de ações, no sentido de preenchimento mais adequado, de modo a proporcionar melhoria no desenvolvimento de futuras pesquisas. Pode-se citar também o tipo de estudo, uma vez que o delineamento transversal não permite o estabelecimento da relação de risco. Como limitação, podem-se mencionar também o período e a população restrita, que influencia, diretamente, os resultados e intervalos de confiança.

\section{CONCLUSÃO E IMPLICAÇÕES PARA A PRÁTICA}

A mortalidade foi de $12,8 \%$, pacientes idosos, com comorbidades cardiovasculares, apresentaram maior chance de óbito. Os principais sintomas foram tosse, dispneia, febre e mialgia, e um terço dos pacientes necessitou de tratamento intensivo, além de antibióticos, anticoagulantes e antiviral associado ao suporte ventilatório. As principais complicações foram hipóxia, insuficiência renal e infecção secundária. Os resultados de um dos centros de referência na pandemia possibilitam discutir medidas epidemiológicas adotadas, com ênfase em conceitos restritivos nos primeiros meses da pandemia.

\section{CONTRIBUIÇÕES DOS AUTORES}

Desenho do estudo. Leticia Pontes. Mitzy Tannia Reichembach Danski. Jéssica de Fátima Gomes Pereira. Letícia Bettega Costa. Andrea Moreira Arrué.

Coleta ou produção dos dados. Simone Martins Nascimento Piubello. Jéssica de Fátima Gomes Pereira Letícia Bettega Costa. Juliana de Oliveira dos Santos.

Análise de dados. Simone Martins Nascimento Piubello. Jéssica de Fátima Gomes Pereira. Leonardo Bigolin Jantsch. Andrea Moreira Arrué.

Interpretação dos resultados. Simone Martins Nascimento Piubello. Jéssica de Fátima Gomes Pereira. Leonardo Bigolin Jantsch. Andrea Moreira Arrué.

Redação e revisão crítica do manuscrito. Leticia Pontes. Mitzy Tannia Reichembach Danski. Simone Martins Nascimento Piubello. Jéssica de Fátima Gomes Pereira. Leonardo Bigolin Jantsch. Letícia Bettega Costa. Juliana de Oliveira dos Santos. Andrea Moreira Arrué.

Aprovação da versão final do artigo. Leticia Pontes. Mitzy Tannia Reichembach Danski. Simone Martins Nascimento Piubello. Jéssica de Fátima Gomes Pereira. Leonardo Bigolin Jantsch. Letícia Bettega Costa. Juliana de Oliveira dos Santos. Andrea Moreira Arrué.

Responsabilidade por todos os aspectos do conteúdo e a integridade do artigo publicado. Leticia Pontes. Mitzy Tannia Reichembach Danski. Simone Martins Nascimento Piubello. Jéssica de Fátima Gomes Pereira. Leonardo Bigolin Jantsch. Letícia Bettega Costa. Juliana de Oliveira dos Santos. Andrea Moreira Arrué.

\section{EDITOR ASSOCIADO}

Antonio José de Almeida Filho (1)

\section{EDITOR CIENTÍFICO}

\author{
Ivone Evangelista Cabral (D)
}

\section{REFERÊNCIAS}

1. Park JJH, Mogg R, Smith GE, Nakimuli-Mpungu E, Jehan F, Rayner CR et al. How COVID-19 has fundamentally changed clinical research in global health. Lancet Glob Health. 2021;9(5):e711-20. http://dx.doi. org/10.1016/S2214-109X(20)30542-8. PMid:33865476.

2. Candido DS, Claro IM, de Jesus JG, Souza WM, Moreira FRR, Dellicour S et al. Evolution and epidemic spread of SARS-CoV-2 in Brazil. Science. 
2020;369(6508):1255-60. http://dx.doi.org/10.1126/science.abd2161. PMid:32703910.

3. Ministério da Saúde (BR). Painel Coronavírus [Internet]. Brasília: Ministério da Saúde; 2021 [cited Feb 16, 2021]. Disponível em: https:// covid.saude.gov.br/

4. Baqui P, Bica I, Marra V, Ercole A, van der Schaar M. Ethnic and regional variations in hospital mortality from COVID-19 in Brazil: a cross-sectional observational study. Lancet Glob Health. 2020;8(8):e1018-26. http:// dx.doi.org/10.1016/S2214-109X(20)30285-0. PMid:32622400.

5. Curitiba. Secretaria Municipal de Saúde. Números COVID-19 [Internet]. Curitiba: Secretária Municipal de Saúde; 2021 [cited Jun 114, 2021]. Disponível em: https://coronavirus.curitiba.pr.gov.br/

6. Alves L. Brazilian ICUs short of drugs and beds amid COVID-19 surge. Lancet. 2021;397(10283):1431-2. http://dx.doi.org/10.1016/S01406736(21)00836-9. PMid:33865483.

7. Pollock AM, Clements L, Harding-Edgar L. Covid-19: why we need a national health and social care service. BMJ. 2020;369:m1465. http:// dx.doi.org/10.1136/bmj.m1465. PMid:32291259.

8. Palaiodimos L, Kokkinidis DG, Li W, Karamanis D, Ognibene J, Arora $S$ et al. Severe obesity, increasing age and male sex are independently associated with worse in-hospital outcomes, and higher in-hospital mortality, in a cohort of patients with COVID-19 in the Bronx, New York. Metabolism. 2020;108:154262. http://dx.doi.org/10.1016/j. metabol.2020.154262. PMid:32422233.

9. Ministério da Saúde (BR). Guia de Vigilância Epidemiológica Emergência de Saúde Pública de Importância Nacional pela Doença pelo Coronavírus 2019. Vigilância de Síndromes Respiratórias Agudas COVID-19 [Internet] Brasília; 2021 [citado 2021 jan 5]. Disponível em: https://portalarquivos. saude.gov.br/images/af_gvs_coronavirus_6ago20_ajustes-finais-2.pdf

10. Secretária Municipal de Saúde. Veja ações do município no combate ao coronavírus, Curitiba (PR) [Internet]. Curitiba; 2020 [citado 2021 maio 6]. Disponível em: https://www.curitiba.pr.gov.br/noticias/vejaacoes-do-municipio-no-combate-ao-coronavirus/55604

11. Secretária Municipal de Saúde. Curitiba tem a menor incidência de COVID-19 entre as maiores capitais, Curitiba (PR). [Internet]. Curitiba 2020 [citado 2021 maio 6]. Disponível em: https://www.curitiba.pr.gov. $\mathrm{br} /$ noticias/curitiba-tem-a-menor-incidencia-de-covid-19-entre-asmaiores-capitais/56070

12. Ranzani OT, Bastos LSL, Gelli JGM, Marchesi JF, Baião F, Hamacher $S$ et al. Characterisation of the first 250000 hospital admissions for COVID-19 in Brazil: a retrospective analysis of nationwide data. Lancet Respir Med. 2021;9(4):407-18. http://dx.doi.org/10.1016/S22132600(20)30560-9. PMid:33460571.

13. Sim BLH, Chidambaram SK, Wong XC, Pathmanathan MD, Peariasamy $\mathrm{KM}, \mathrm{Hor} \mathrm{CP}$ et al. Clinical characteristics and risk factors for severe COVID-19 infections in Malaysia: A nationwide observational study. Lancet Reg Health West Pac. 2020;4:e100055. http://dx.doi.org/10.1016/j. lanwpc.2020.100055. PMid:33521741.

14. Mikami T, Miyashita H, Yamada T, Harrington M, Steinberg D, Dunn A et al. Risk factors for mortality in patients with COVID-19 in New York city. J Gen Intern Med. 2021;36(1):17-26. http://dx.doi.org/10.1007/ s11606-020-05983-z. PMid:32607928.

15. Grupta S, Hayek SS, Wang W, Chan L, Mathews KS, Melamed ML, et al. Factors associated with death in critically III patients with Coronavirus Disease 2019 in the US. JAMA Intern Med. 2020;180:e11. http://dx.doi. org/10.1001/jamainternmed.2020.3596.

16. Ministério da Saúde (BR). Secretaria de Atenção Especializada à Saúde. Departamento de Atenção Hospitalar, Domiciliar e de Urgência. Protocolo de Tratamento do Novo Coronavírus (2019-nCoV) [Internet]
Brasília: Ministério da Saúde; 2021 [cited Jan 5, 2021]. Disponível em: https://portalarquivos2.saude.gov.br/images/pdf/2020/fevereiro/05/ Protocolo-de-manejo-clinico-para-o-novo-coronavirus-2019-ncov.pdf

17. Menni C, Valdes AM, Freidin MB, Sudre CH, Nguyen LH, Drew DA et al Real-time tracking of self-reported symptoms to predict potential COVID-19. Nat Med. 2020;26(7):1037-40. http://dx.doi.org/10.1038/ s41591-020-0916-2. PMid:32393804.

18. Sanyaolu A, Okorie C, Marinkovic A, Patidar R, Younis K, Desai P et al Comorbidity and its Impact on Patients with COVID-19. SN Compr Clin Med. 2020;2(8):1-8. http://dx.doi.org/10.1007/s42399-020-00363-4. PMid:32838147.

19. Ministério da Saúde (BR). Secretaria de Vigilância em Saúde. Doença pelo Coronavírus COVID-19. Boletim Epidemiológico Especial. Semana Epidemiológica 52 (20 a 26/12/2020) [Internet]. Brasília: Ministério da Saúde; 2020 [citado 2021 jan 5]. Disponível em: https://www.gov.br/ saude/pt-br/media/pdf/2020/dezembro/30/boletim_epidemiologico_ covid_43_final_coe.pdf

20. Morales DR, Conover MM, You SC, Pratt N, Kostka K, Duarte-Salles Tet al. Renin-angiotensin system blockers and susceptibility to COVID-19: an international, open science, cohort analysis. Lancet Digit Health 2021;3(2):e98-114. http://dx.doi.org/10.1016/S2589-7500(20)30289-2. PMid:33342753.

21. Centers for Disease Control and Prevention. COVID-NET [Internet] New York: CDC; 2021 [citado 2021 jan 5]. Disponível em: https://gis. cdc.gov/grasp/COVIDNet/COVID19_5.html

22. Hirsch JS, Ng JH, Ross DW, Sharma P, Shah HH, Barnett RL et al Acute kidney injury in patients hospitalized with COVID-19. Kidney Int. 2020;98(1):209-18. http://dx.doi.org/10.1016/j.kint.2020.05.006. PMid:32416116.

23. Li W, Lin F, Dai M, Chen L, Han D, Cui $Y$ et al. Early predictors for mechanical ventilation in COVID-19 patients. Ther Adv Respir Dis. 2020;14:1753466620963017. http://dx.doi.org/10.1177/1753466620963017. PMid:33054630.

24. Furtado RHM, Berwanger O, Fonseca HA, Corrêa TD, Ferraz LR, Lapa $M G$ et al. Azithromycin in addition to standard of care versus standard of care alone in the treatment of patients admitted to the hospital with severe COVID-19 in Brazil (COALITION II): a randomised clinical trial. Lancet. 2020;396(10256):959-67. http://dx.doi.org/10.1016/S01406736(20)31862-6. PMid:32896292.

25. Hanif A, Khan S, Mantri N, Hanif S, Saleh M, Alla Y et al. Thrombotic complications and anticoagulation in COVID-19 pneumonia: a New York City hospital experience. Ann Hematol. 2020;99(10):2323-8. http:// dx.doi.org/10.1007/s00277-020-04216-x. PMid:32808105.

26. Centers for Disease Control and Prevention. Older adults [Internet] New York: CDC; 2021 [citado 2021 jan 5]. Disponível em: https://www. cdc.gov/coronavirus/2019-ncov/need-extra-precautions/older-adults. $\mathrm{html}$

27. Nikolich-Zugich J, Knox KS, Rios CT, Natt B, Bhattacharya D, Fain MJ. SARS-CoV-2 and COVID-19 in older adults: what we may expect regarding pathogenesis, immune responses, and outcomes. Geroscience. 2020;42(2):505-14. http://dx.doi.org/10.1007/s11357-020-00186-0. PMid:32274617.

28. Souza ZA Fo, Nemer CRB, Teixeira E, Neves ALM, Nascimento MHM, Medeiros HP, et al. Factors associated with coping with the COVID-19 pandemic by older adults with comorbidities. Esc Anna Nery. 2021;25(spe):e20200495. http://dx.doi.org/10.1590/2177-9465ean-2020-0495. 